\author{
Military Technical College \\ Kobry El-Kobbah, \\ Cairo, Egypt
}

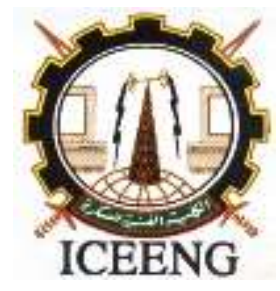

\author{
$11^{\text {th }}$ International Conference \\ on Electrical Engineering \\ ICEENG 2018
}

\title{
SIMULATION OF TRADITIONAL AND BALANCED OPTICAL CODE DIVISION MULTIPLE ACCESS RECEIVERS
}

\author{
M. S. Hemdan*, T. M. Bazan**, K. M. Hassan ${ }^{\star * *}$
}

\begin{abstract}
Optical code division multiple access (OCDMA) is an encouraging multiplexing technique for future optical communication systems. This paper analyzes the performance of the traditional and balanced OCDMA receivers associated with their appropriate codes. Several 2-D codes with different properties are simulated in this work. The experimental simulations are implemented by Optisystem software to demonstrate the performance of a three-user OCDMA system operating at $1 \mathrm{~Gb} / \mathrm{s}$. The simulation results reveal that the traditional receiver employing zero-correlation codes has better performance if compared to the balanced scheme. In addition, the assigned wavelengths to each user highly affect the system performance.
\end{abstract}

\section{KEY WORDS}

Optical Code Division Multiplexing, Simulation, Optical Codes, Balanced Receiver

\section{Introduction}

An optical code division multiple access (OCDMA) system is regarded as an auspicious technique in optical fiber communication networks [1-3]. The key OCDMA advantage is to support a large number of subscribers in an asynchronous way. In OCDMA systems, each user is allocated a specific code and the data from all active users in the network is sent through the optical fiber. At the intended receiver, the desired signal is extracted through the decoder that stores the relevant code. However, the utilized code and the receiver configuration play significant roles in the system performance. In fact, the traditional and the balanced receivers are considered the basic OCDMA receiver configurations in the literature [4-6]. In the last two decades, most studies have mathematically investigated the performance analysis of different OCDMA receivers utilizing different code types.

\footnotetext{
* Dept. of Electrical Engineering, Suez Canal University, Egypt.

** Egyptian Armed Forces.

*** Dept. of Electrical Engineering, Future University, Egypt.
} 
In fact, the simulation is an effective method used to investigate the OCDMA system performance. The earlier OCDMA simulation efforts have guided to many directions; e.g., OCDMA over wavelength division multiplexing [7], spectral amplitude coding [8], 2-D codes [9-11], radio over fiber-OCDMA [12], NRZ/RZ modulation format [13], and security employing OCDMA [14].

In this paper, the simulations of both traditional and balanced OCDMA receivers are executed by the Optisystem 12. In addition, the study includes three code families with different properties namely, multi-diagonal (MD) [10], random diagonal (RD) [11], and Hadamard codes (HC) [15]. The main OCDMA code properties and different OCDMA receiver configurations are introduced in section 2. Section 3 presents the simulation setup of the traditional and balanced OCDMA receivers with their compatible codes. Section 4 presents the simulation result and finally, Section 5 outlines the main conclusions.

\section{OCDMA CODE PROPERTY}

Optical codes take the form of a carefully designed sequence of zeros and ones; produced at a chip rate that is higher than the baseband data rate. An OCDMA code $\mathrm{C}\left(N, w, \lambda_{\mathrm{a}}, \lambda_{c}\right)$ is a set of ones and zeros sequences where $N$ is the length of the codeword, $w$ is the code weight representing the number of active chips in every codeword, $\lambda_{\mathrm{a}}$ is value of the auto-correlation and $\lambda_{c}$ is the value of the crosscorrelation. One-dimensional (1-D) codes are direct spread either in time or in wavelength domain whereas the two-dimensional (2-D) codes are spread in both time and wavelength domains. However, 2-D codes give higher cardinality with better performance than 1-D codes [4]. Nevertheless, the codes employed in any OCDMA system should have a good correlation property to satisfy the code orthogonality condition. MD and RD are recent examples of codes whose $\lambda_{c}=0$ and 1, respectively, and can be utilized with the traditional receiver Fig.1. On the other hand, although their $\lambda_{c}=N / 4$, HCs can be used with the balanced receiver Fig. 2 in order to eliminate the multiple access interference (MAI). In the traditional receiver, only one branch is utilized including the OCDMA decoder and photodetector whereas the balanced receiver is comprised of two complementary branches. One of such the two branches is used to retrain the desired data while the other to cancel the MAl. Table 1 summarizes the main properties of the used codes.

Table 1. The main properties of the used codes

\begin{tabular}{|c|c|c|c|}
\hline Property & MD & RD & HC \\
\hline Code length & $N=K \times w$ & $N=K+2 w-3$ & $\mathrm{~N}=2^{\mathrm{m}}, m=2,3,4, \ldots$ \\
\hline Code weight & $w$ & $w$ & $w=N / 2$ \\
\hline Cross-correlation value & 0 & 1 & $N / 4$ \\
\hline Code cardinality & $K$ & $K$ & $K=N-1$ \\
\hline No. of used wavelength & $N$ & $N$ & $N$ \\
\hline
\end{tabular}


Table 2. MD code structure for 3 users when $\mathrm{w}=2$

\begin{tabular}{|c|c|c|c|c|c|c|}
\hline \multirow{2}{*}{$\begin{array}{c}\text { User } \\
\text { No. }\end{array}$} & \multicolumn{6}{|c|}{ Code sequences $(6,2,2,0)$} \\
\cline { 2 - 7 } & $t_{1}$ & $t_{2}$ & $t_{3}$ & $t_{4}$ & $t_{5}$ & $t_{6}$ \\
\hline $\mathbf{1}$ & $\lambda_{5}$ & 0 & 0 & 0 & 0 & $\lambda_{2}$ \\
\hline $\mathbf{2}$ & 0 & $\lambda_{6}$ & 0 & 0 & $\lambda_{3}$ & 0 \\
\hline 3 & 0 & 0 & $\lambda_{4}$ & $\lambda_{1}$ & 0 & 0 \\
\hline
\end{tabular}

Table 3. RD code structure for 3 users when $\mathrm{w}=3$

\begin{tabular}{|c|c|c|c|c|c|c|c|}
\hline \multirow{2}{*}{$\begin{array}{c}\text { User } \\
\text { No. }\end{array}$} & \multicolumn{7}{|c|}{ Code sequences $(\mathbf{7}, \mathbf{3}, \mathbf{3}, \mathbf{1})$} \\
\cline { 2 - 8 } & $t_{1}$ & $t_{2}$ & $t_{3}$ & $t_{4}$ & $t_{5}$ & $t_{6}$ & $t_{7}$ \\
\hline 1 & 0 & 0 & 0 & $\lambda_{4}$ & $\lambda_{5}$ & $\lambda_{6}$ & 0 \\
\hline 2 & 0 & 0 & $\lambda_{3}$ & 0 & 0 & $\lambda_{6}$ & $\lambda_{7}$ \\
\hline 3 & 0 & $\lambda_{2}$ & 0 & 0 & $\lambda_{5}$ & 0 & $\lambda_{7}$ \\
\hline
\end{tabular}

Table 4. $\mathrm{HC}$ structure for 3 users when $\mathrm{w}=2$

\begin{tabular}{|c|c|c|c|c|}
\hline \multirow{2}{*}{$\begin{array}{c}\text { User } \\
\text { No. }\end{array}$} & \multicolumn{4}{|c|}{ Code sequences $(\mathbf{4 , 2}, \mathbf{2}, \mathbf{1})$} \\
\cline { 2 - 5 } & $t_{1}$ & $t_{2}$ & $t_{3}$ & $t_{4}$ \\
\hline $\mathbf{1}$ & $\lambda_{1}$ & 0 & $\lambda_{3}$ & 0 \\
\hline $\mathbf{2}$ & $\lambda_{1}$ & $\lambda_{2}$ & 0 & 0 \\
\hline $\mathbf{3}$ & $\lambda_{1}$ & 0 & 0 & $\lambda_{4}$ \\
\hline
\end{tabular}

\section{SIMULATION SETUP}

The simulation of an OCDMA system is carried-out using the simulation software "OptiSystem" version 12. OptiSystem is a comprehensive software design suite that enables users to plan, test, and simulate optical links in the transmission layer of modern optical networks. The simulation utilizes a laser diode at a bit rate of $1 \mathrm{Gbps}$ and ITU-T G.652 single-mode optical fiber (SMF). The parameters of the proposed simulation setup are presented in Table 5 .

Table 5. Parameters used in simulation setup

\begin{tabular}{|c|c|}
\hline Parameter & Value \\
\hline Bit rate & $1 \mathrm{Gbps}$ \\
\hline Signal format & $\mathrm{NRZ}$ \\
\hline Fiber attenuation & $0.25 \mathrm{~dB} / \mathrm{km}$ \\
\hline Fiber dispersion & $18 \mathrm{ps} / \mathrm{nm} / \mathrm{km}$ \\
\hline Fiber length & $40 \mathrm{~km}$ \\
\hline Dark current & $10 \mathrm{nA}$ \\
\hline Number of users & 3 \\
\hline Transmitted power & $-10 \mathrm{dBm}$ \\
\hline Operating wavelength & $1550 \mathrm{~nm}$ \\
\hline
\end{tabular}

The simulation model includes three parts; transmitter, fiber link and receiver sections. The transmitter section consists of four components; pulse pattern generator, non-return-zero (NRZ) pulse generators, laser array associated with 
external modulators. In the beginning, the useful binary data signals are generated by the pseudo-random binary sequence with the NRZ line coding. Then, these signals are fed to the Mach-Zehnder modulator to modulate the intensity of the optical CW laser signals. Therefore, the optical signals transmitted from all active users will be combined and broadcasted through the SMF. In the receiver module, the incoming optical signal is distributed by the fiber Bragg grating (FBG) decoder that has the same codeword of the intended encoder. Then, the user signal is detected by the photodetector (PD) and the original data are filtered and restored using a low pass filter (LPF). The simulation arrangement of the OCDMA traditional and balanced receivers for each user is shown in Fig.3 and Fig.4, respectively.

Note that since the code weight is two, two reflective FBGs are employed in the receiver to simulate the codeword in the intended encoder. For the balanced receiver, the decoder in the lower branch has the code complement of the upper one. The entire OCDMA simulation layout of the three-user system using the traditional receiver with MD code sequence is described in Fig.5.

\section{SIMULATION RESULTS}

The performance analysis of a three-user OCDMA system for both traditional and balanced receivers is analyzed. Fig. 6 shows the original input data which is NRZ pulse format. Fig.7 shows the received MD signal of user no. 1 after passing through the LPF. The noise signal is due to the conventional noise sources (shot, thermal, dark current, etc.).

Fig. 8 shows the wavelength spectrum that consists of the combined six wavelengths of the three users utilized the MD code family at the beginning of the optical fiber. The six wavelengths are $1548.5 \mathrm{~nm}, 1549.1 \mathrm{~nm}, 1549.7 \mathrm{~nm}, 1550.3 \mathrm{~nm}, 1550.9 \mathrm{~nm}$, and $1551.5 \mathrm{~nm}$. Fig.9 shows the optical signal spectrums for the user no. 1 at the output of the traditional receiver decoder. It is shown that the peak received power $(\sim-54 \mathrm{dBm})$ occurs at the assigned wavelengths of user no. $1 \quad\left(\lambda_{2}=1549.1 \mathrm{~nm}\right.$, $\lambda_{5}=1550.9 \mathrm{~nm}$ ) as indicated in table 1. Fig.10 and Fig.11 show the corresponding optical spectrum for the user no. 2 and 3 , respectively.

Using the traditional receiver with $\mathrm{RD}(w=3)$ and $\mathrm{MD}(w=2)$ codes for transfer data from user no. 2 over $40 \mathrm{~km}$ distance has been considered. The estimated performance for the two cases in terms of BER has been checked using the eye diagram. It is found that the performance of the traditional receiver with MD codes gives better bit error rate (minimum $B E R=5.7 \times 10^{-9}$ ) if it is compared to that of $R D$ (minimum BER of $5.6 \times 10^{-3}$ ) as shown in Fig.12 and Fig.13, respectively. This is, of course, due to the zero cross-correlation of the MD codes instead of one as the case of $\mathrm{RD}$. For the RD case, the close eye diagram shown in Fig.12 is due to the level MAI is one-third of the code weight. However, increasing the code weight will enhance the performance. 
Fig. 14 shows the eye diagram of the received data from the balanced receiver using HCs for the user no. 2. In this case, the BER of $2.9 \times 10^{-7}$ has been achieved. Comparing this value with that estimated from Fig.12 $\left(5.7 \times 10^{-9}\right)$, it is clear that the performance for MD code is better. In the case of balanced configuration, the MAI is canceled but there is a 3-dB loss due to the presence of the two branches and, consequently, the power of the received useful signal is halved. Table 6 gives a comparison between Traditional and balanced receivers at fiber length $L=40 \mathrm{~km}$.

Table 6. OCDMA Receivers Comparison at fiber length $\mathrm{L}=40 \mathrm{~km}$

\begin{tabular}{|c|c|c|c|}
\hline Receivers & \multicolumn{2}{|c|}{$\begin{array}{c}\text { Traditional } \\
\text { receiver }\end{array}$} & $\begin{array}{c}\text { Balanced } \\
\text { receiver }\end{array}$ \\
\hline Codes & $\mathrm{MD}$ & $\mathrm{RD}$ & $\mathrm{HC}$ \\
\hline $\mathrm{BER}$ & $5.7 \times 10^{-9}$ & $5.6 \times 10^{-3}$ & $2.9 \times 10^{-7}$ \\
\hline
\end{tabular}

Finally, Fig. 15 shows full comparison between the Traditional and balanced receivers utilizing its appropriate codes.

To study the effect of fiber length, the simulation is executed for different transmission lengths when $\alpha=0.25 \mathrm{~dB} / \mathrm{Km}$ and $D=18 \mathrm{ps} / \mathrm{nm} / \mathrm{km}$. as depicted in Fig. 16. Increasing the fiber transmission distance will also increase the BER. In fact, increasing the optical fiber length leads to a decrease in the received optical signal, degrading the system performance. Actually, several factors are also affecting the system performance including the attenuation, absorption, and dispersion. Similar trends are observed for three users. It is also noted that user no. 1 has the worst performance since the assigned wavelengths of such a user (refer to Table 2) are far from the central wavelength $(1.55 \mu \mathrm{m})$; undergoing more losses and dispersion values. On the other hand, choosing a code weight with allocated wavelengths near $1.55 \mu \mathrm{m}$ (e.g., the user no. 3 in which $\lambda_{1}=1548.5 \mathrm{~nm}, \lambda_{4}=1550.3 \mathrm{~nm}$ ) shows a better performance.

Therefore, the performance of OCDMA system is greatly affected by the assigned wavelengths to each user.

\section{Conclusion}

In this paper, the performance of traditional and balanced OCDMA receiver configurations with various codes is presented. The simulated results showed that the traditional receiver utilizing MD codes gives better performance if it is compared to the balanced receiver utilizing the Hadamard codes. It is found also that the performance of the balanced receiver utilizing the HCs is superior to the traditional receiver with $\mathrm{RD}$ codes. Then, the traditional receiver utilized $\mathrm{MD}$ codes is recommended to be employed in the OCDMA systems. Furthermore, the wavelength distribution for each OCDMA code is an additional design parameter which affects strongly the performance of the system.

\section{Figures}




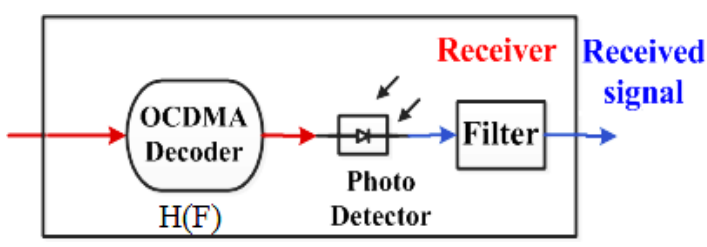

Fig.1. OCDMA Block diagram of the traditional receiver

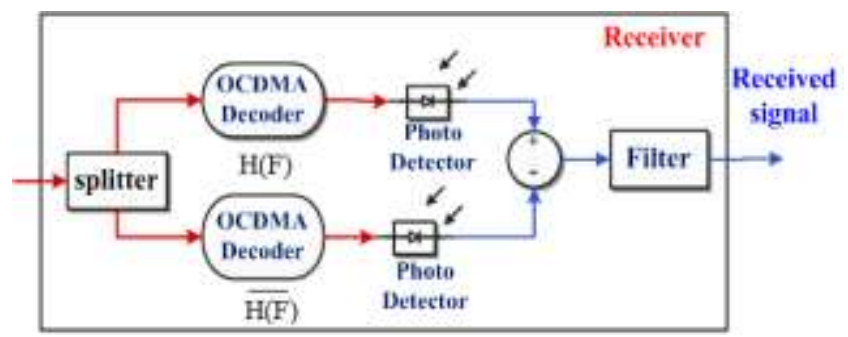

Fig.2. OCDMA Block diagram of the balanced receiver

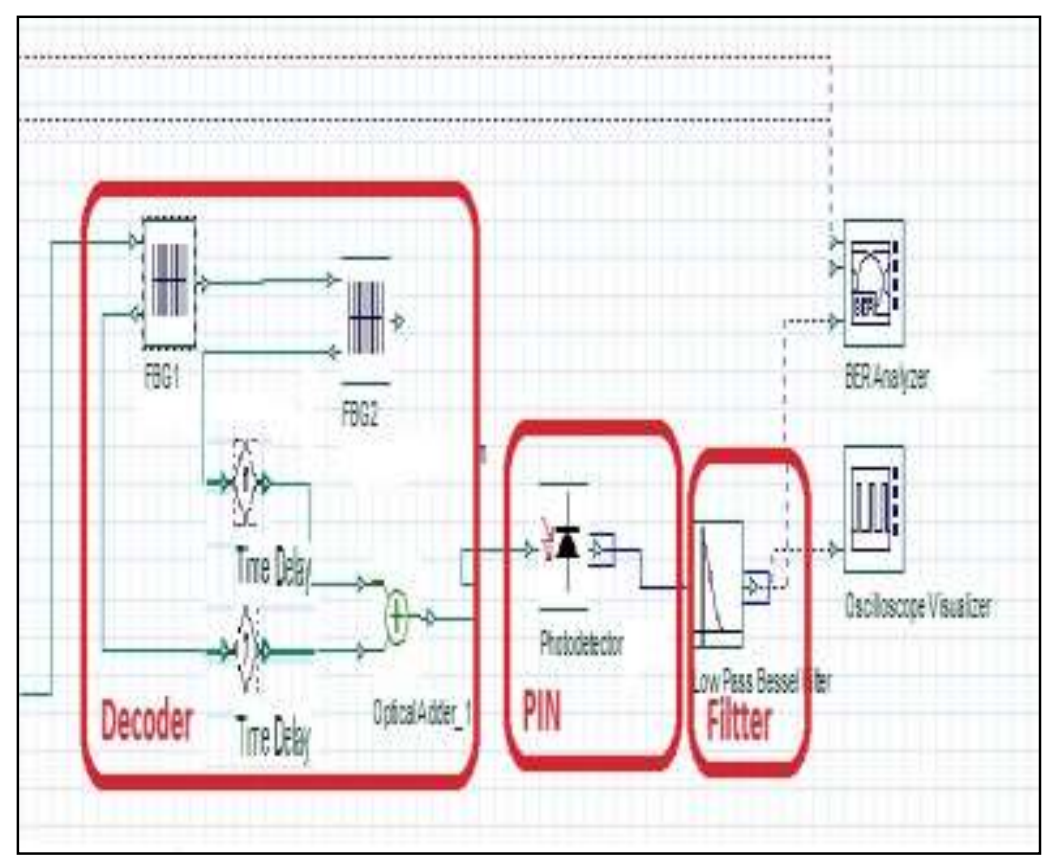

Fig.3. Layout design of OCDMA traditional receiver

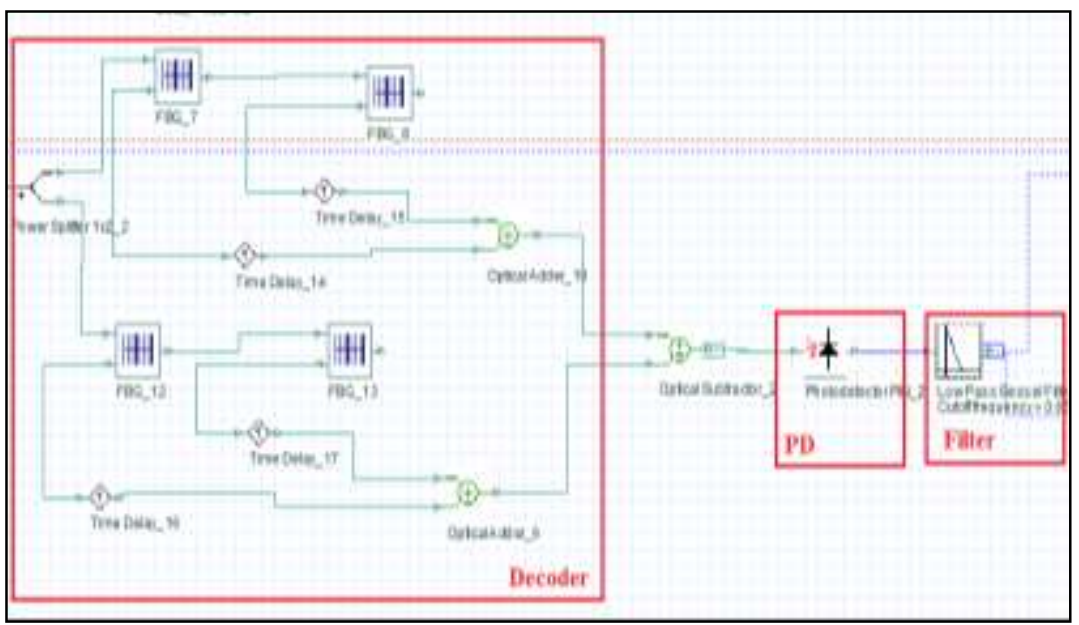

Fig.4. Layout design of OCDMA balanced receiver 


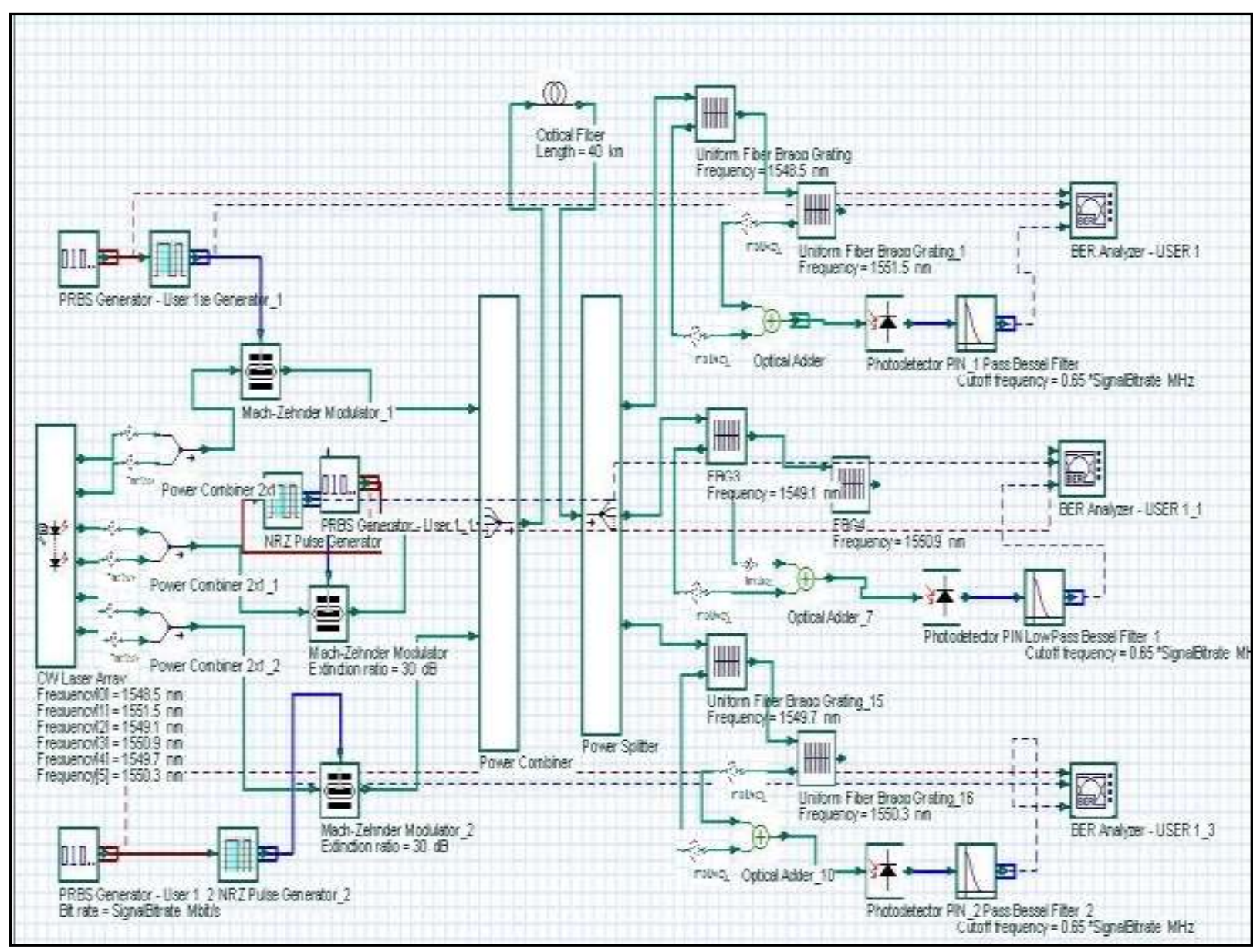

Fig.5. Layout of a three-user OCDMA system with the traditional receiver using MD code family

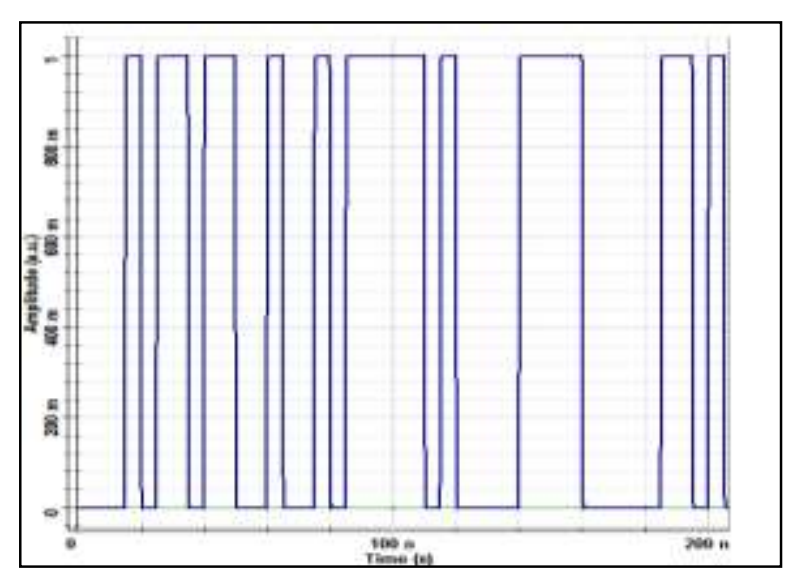

Fig.6. The input electrical signal data

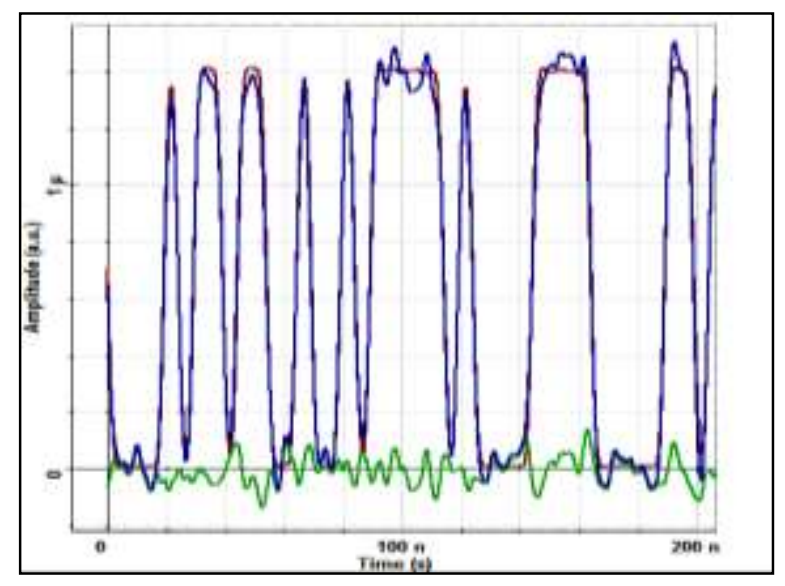

Fig.7. The received waveform of user no. 1 at the output of the LPF 


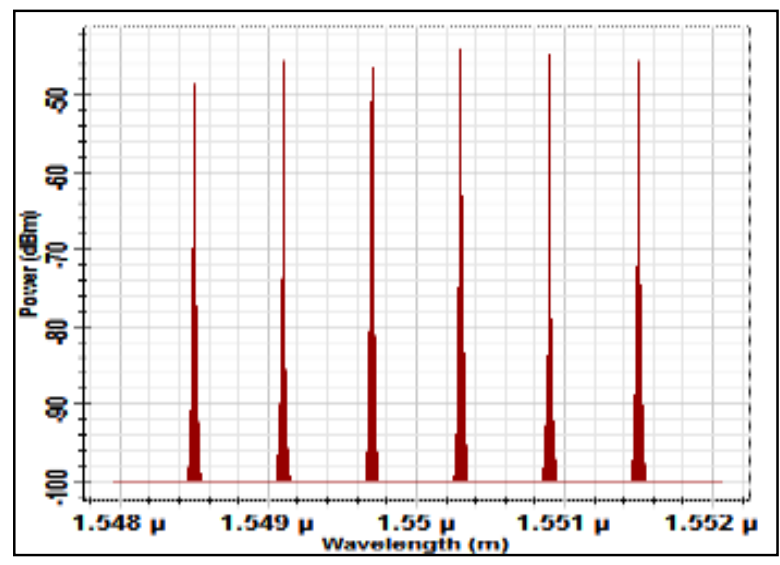

Fig.8. The corresponding wavelength spectrum of the three MD codes

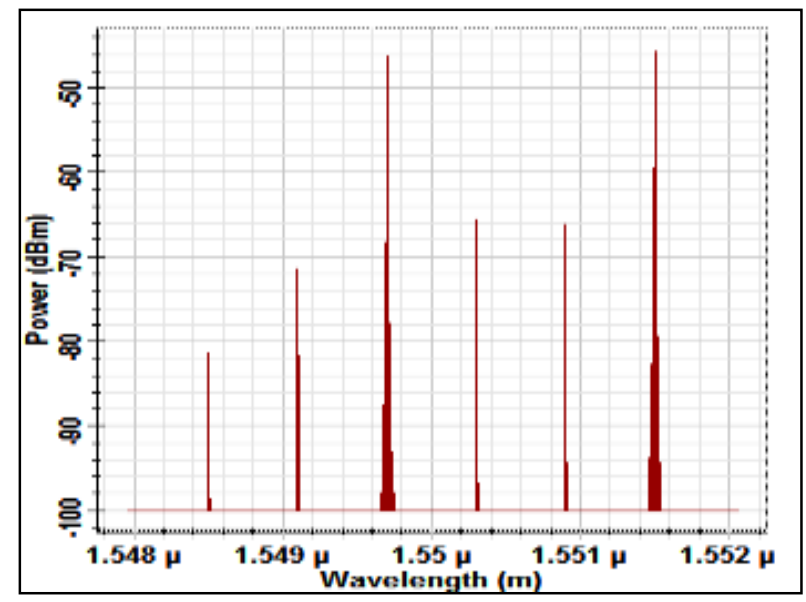

Fig.10. The output spectrum at the receiver side after correlation for User no.2

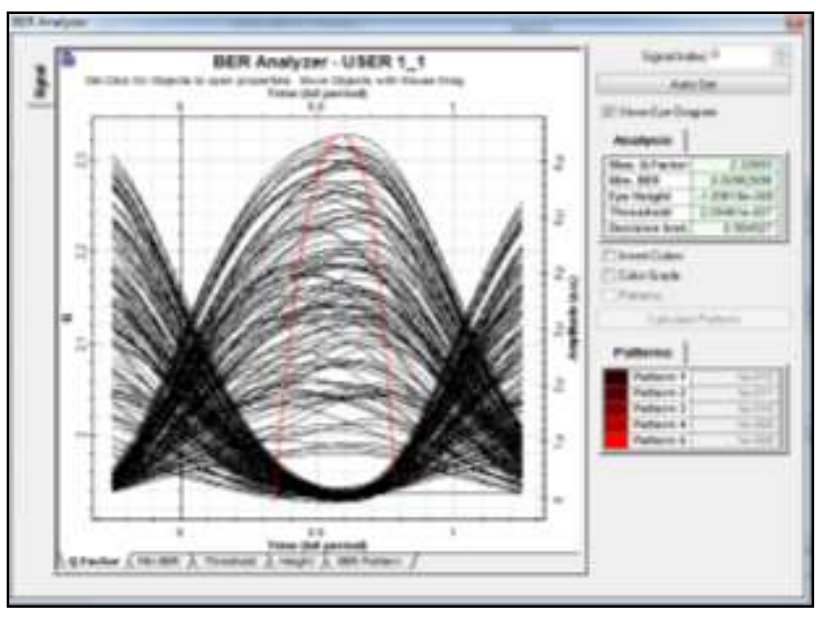

Fig.12. Eye diagrams of the traditional receiver with $\mathrm{RD}$ codes

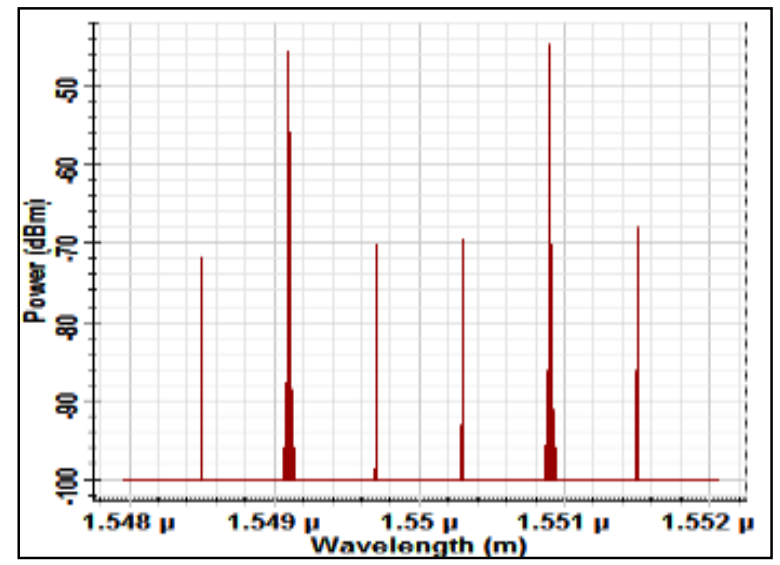

Fig.9. The output spectrum at the receiver side after correlation for User no. 1

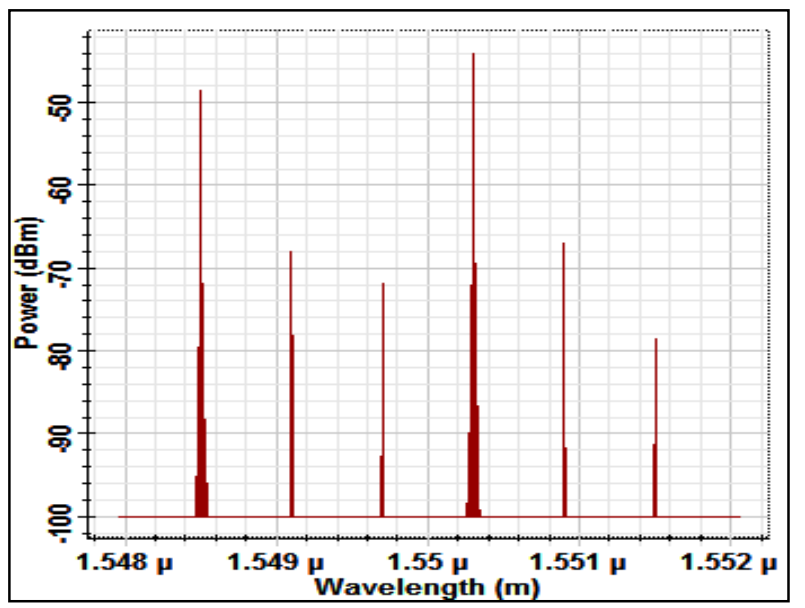

Fig.11. The output spectrum at the receiver side after correlation for User no.3

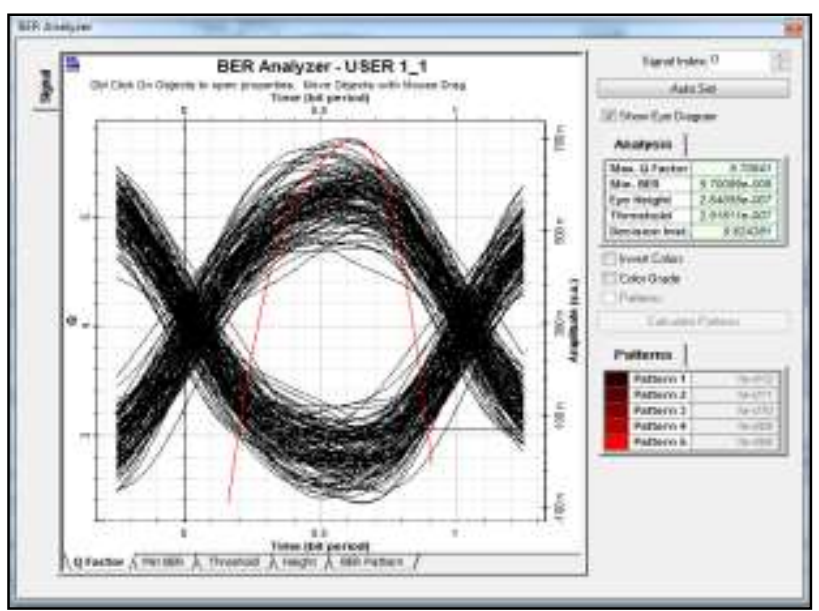

Fig.13. Eye diagrams of the traditional receiver with $M D$ codes 


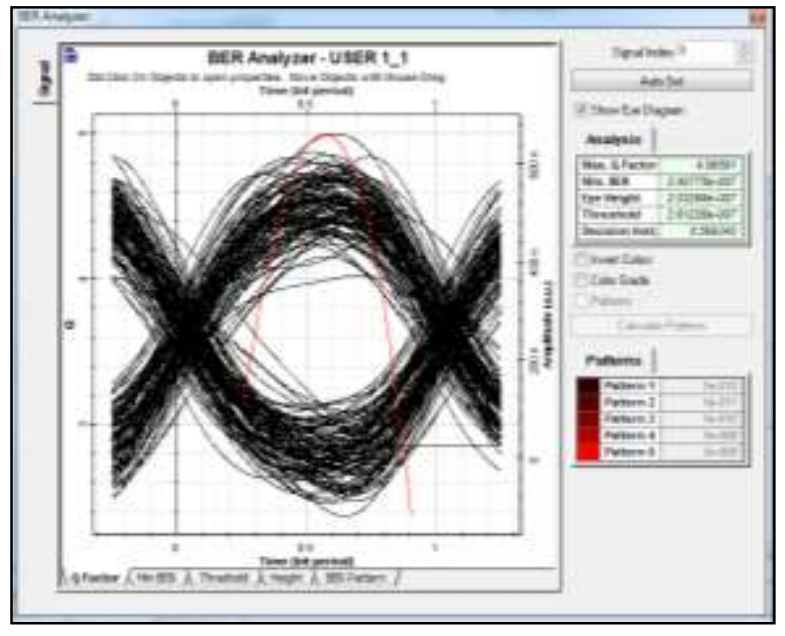

Fig.14. Eye diagram of the balanced receiver with $\mathrm{HCs}$

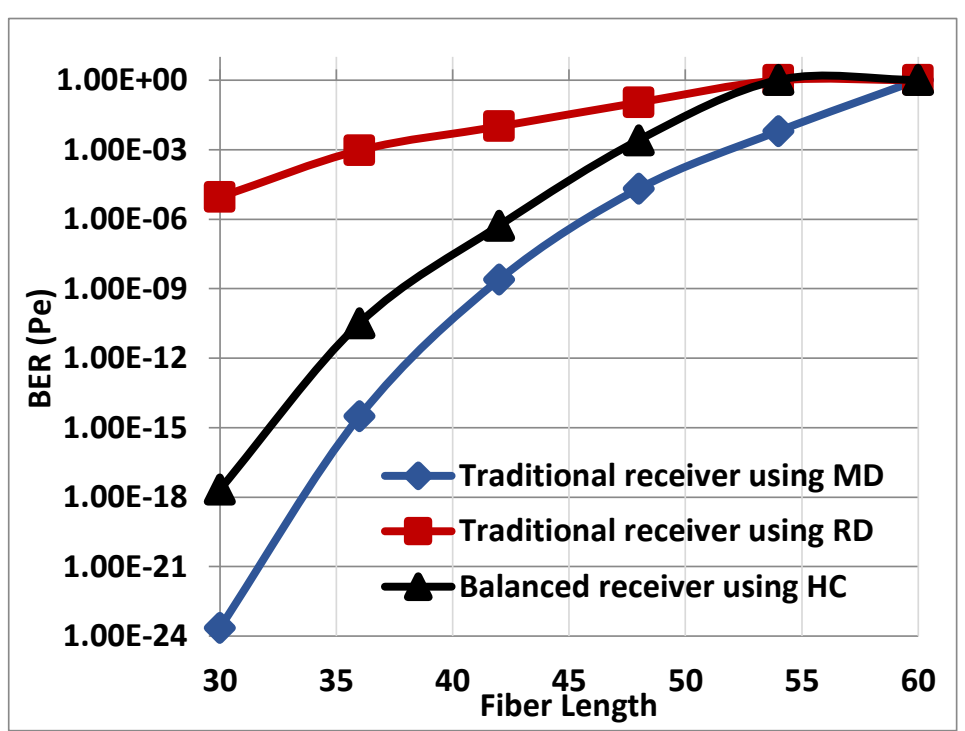

Fig.15. BER versus fiber length for traditional and balanced receivers

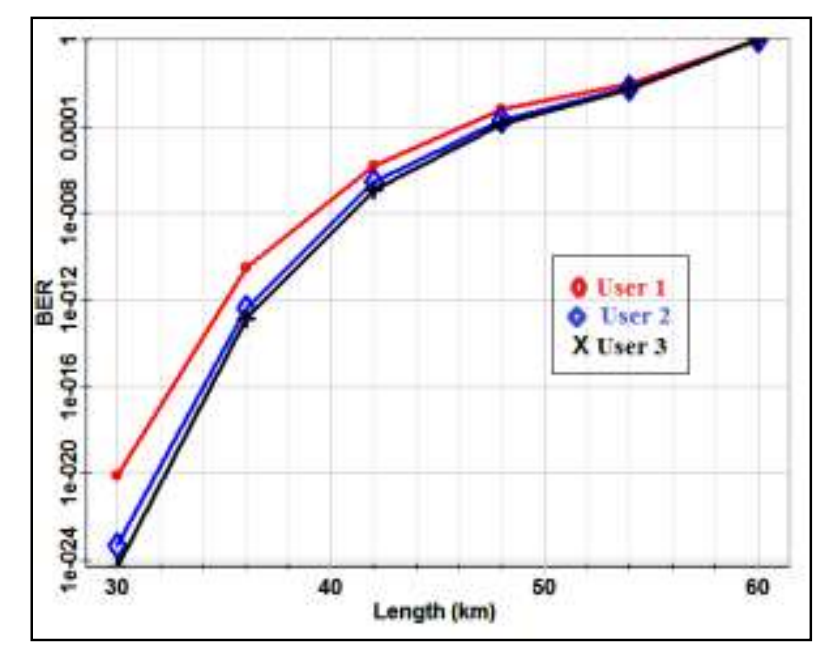

Fig.16. BER versus fiber length for the traditional receiver

\section{Reference}

[1] M. Othman, M. Rejab, R. Talib, N. Cholan, M. Abdullah, S. Aljunid, and M. Abdullah, "Comparison of detection techniques in optical CDMA access network for point to multipoint configuration.", IEEE International Conference on Electronic Design, (2008).

[2] S. Saleh, M. Moghaddasi, and S. Anas, "Variable-weight optical code division multiple access system using different detection schemes." J. of Telecommun. and Information Technology, vol. 3, pp. 50-59 (2016).

[3] M. Hadi, and M. Pakravan, "Analysis and design of adaptive OCDMA passive optical networks," J. Lightw. Technol., vol. 35, pp. 2853 - 2863 (2017).

[4] G.-C. Yang and W. Kwong, "Prime codes with applications to CDMA optical and wireless networks,"Boston, MA: Artech House, (2002). 
[5] X. Wang, N. Wada, T. Miyazaki, G. Cincotti, and K Kitayama "Asynchronous multiuser coherent OCDMA system with code-shift-keying and balanced detection." IEEE Journal of Select. Top. Quan. Elect., vol. 13, pp. 1463 - 1470 (2007).

[6] H. Heo, S. S. Min, Y. Won, Y. Yeon, and B. Kim, "A new family of 2-D wavelengthtime spreading code for optical code multiple access system with balanced detection,"IEEE Photon. Technol. Lett., vol. 16, pp. 2189-2191 (2004).

[7] N. Ahmed, M. Rashid, and M. Islam. "Hybrid OCDMA over WDM system with DPSK modulation using direct and complementary subtraction detection techniques." IEEE Inter. Telecommunication Networks and Applications Conf., (2016).

[8] T. Abd, Thanaa, S. Aljunid, H. Fadhil, R. Ahmad, and M. Rashid, "New approach for evaluation of the performance of spectral amplitude coding-optical code division multiple access system on high-speed data rate." IET Communications, vol. 6, pp. 1742-1749 (2012).

[9] A. Jena and $U$. Bhanja. "Performance analysis of modified two dimensional Golomb code." IEEE Annual India Conference, pp. 1-6 (2016).

[10] T. Hussein, S. Aljunid, H. Fadhil, M. Junita, N. Saad, "Modelling and simulation of a $1.6 \mathrm{~Tb} / \mathrm{s}$ optical system based on multi-diagonal code and optical codedivision multiple-access," Ukr. J. Phys. Opt. vol. 13, pp. 54-66 (2012).

[11] H. Fadhil, S. Aljunid, and B. Ahmed, "Performance of OCDMA systems using random diagonal code for different decoders architecture schemes," The International Arab Journal of Information Technology, vol. 7, pp. 1-5 (2010).

[12] Z. Ibrahim, C. Rashidi, S. Aljunid, A. Rahman, and M. Anuar, "Performance analysis of optical CDMA based on radio over fiber (RoF) technique." IEEE International Conference on Electronic Design (2016).

[13] M. Norazimah, S. Aljunid, H. Al-Khafaji, H. Fadhil, and M. Anuar, "Performance of different SAC-OCDMA detection schemes with NRZ and RZ data formats." IEEE Symposium on Industrial Electronics and Applications (2013).

[14] M. Bharti, A. Sharma, and M. Kumar, "Simulative analysis of 2-code keying approach using Walsh Hadamard codes to enhance security and reduce dispersion in OCDMA system," IEEE International Conference on Data Mining and Intelligent Computing, (2014). 